\title{
Numerical and Experimental Study on the Multiphase Flow in Gas-Carried Powder Injection Desulphurization Processes
}

\author{
Shiyao Lin \\ School of Naval Architecture and Ocean Engineering \\ Huazhong University of Science and Technology \\ Wuhan, China \\ shiyao_lin@hust.edu.com \\ Han Chen \\ School of Civil Engineering and Mechanics \\ Huazhong University of Science and Technology \\ Wuhan, China \\ hanchen@hust.edu.cn
}

\author{
De Xie \\ School of Naval Architecture and Ocean Engineering \\ Huazhong University of Science and Technology \\ Wuhan, China \\ dexie@hust.edu.com
}

\begin{abstract}
Injection of gas-carried powders by an immersed lance is among the most popular desulphurization methods in many metallurgical processes. This paper proposes a CFD algorithm to simulate the transient multiphase flow in the powder injection process, using the volume of fluid (VOF) method to calculate the gas-liquid flow and discrete phase model (DPM) to capture particles motions. The $k-\varepsilon$ model is also adopted to solve the turbulent flow field. Experimental results on a water model are compared with CFD simulation results and qualitative agreement is found. Then, the CFD algorithm is used to numerically study the multiphase flow in the powder injection process, with an emphasis on the particles distribution. Influences on powders distribution of lance entering length and injection speed are also investigated.
\end{abstract}

Keywords- desulphurization; CFD; multiphase flow; VOF; DPM.

\section{INTRODUCTION}

In response to the demand for high quality iron and steel to construct gigantic structures, produce delicate instruments and manufacture powerful equipments. People nowadays are attaching more and more importance to the treating of molten iron prior to continuous casting, of which desulphurization is a necessary part. Since the 1970's, ladle metallurgy has been practiced as an universe technique for treating molten steel [1], and therefore, researchers began to grow interest in it. At first, experimental studies were dominant, and one of the most significant one was carried out by Sinha and McNallan to detect mixing of particles in water using electrodes [2]. Another form was to utilize the high speed cameras. The representative ones are that Ozawa and Mori tried to understand particle penetration through bubbles [3] and that Torri and Yang studied bubble motion inside gasstirred ladles [4]. A more advanced method is to use the Laser Doppler Anemometer (LDA) to display the distribution accurately, which was adopted by Morud and Hjertager to detect particles in a bioreactor [5]. In general, although there are at least three ways to conduct the experiments, the water model experiment is the most welcomed one, for its simplicity and economy, while preserving enough accuracy. In this paper, water model experiments are practiced with a camera to record flow inside a cylindrical tank.

From the 1980's, with the development of CFD simulation, numerical approaches have been proposed and promoted. Among these studies, the k- $\varepsilon$ turbulent model has been the most widely employed one. Furthermore, Mazumdar and Guthrie did some amendment to coefficients of the k- $\varepsilon$ model to attain higher accuracy [6]. Based on the dynamic fluid model, Venturini and Goldschmit proposed a mass transfer model to investigate gas-liquid reactions [7]. To understand flows in actual ladles better, Xia carried out CFD calculation and experiments on flows in Wood's metal, whose density and viscosity are more close to molten iron [8]. Nevertheless, these works mostly focused on the gas-liquid flow, and the studies addressing particles motions and distribution in ladles are sparse.

In this paper, the $\mathrm{k}-\varepsilon$ model is adopted to study the flow and the VOF method is used to determine the free surfaces. In order to investigate particles evolution, the DPM is employed in a coupled manner. CFD calculations and experiments are practiced on water model first and the real ladle flow is simulated numerically. The results mostly focus on the particles distribution and motion. Influences of parameters such as inlet speed on the distribution are studied.

\section{Methodology OF CFD ALGORITHM}

\section{A. Basic fluid dynamic equations}

Wherever Times is specified, Times Roman or Times New Roman may be used. If neither is available on your word processor, please use the font closest in appearance to Times. Avoid using bit-mapped fonts if possible. True- 
Type 1 or Open Type fonts are preferred. Please embed symbol fonts, as well, for math, etc.

Gas-stirred fluid flow follows the mass conservation law in equation (1):

$$
\frac{\partial \rho}{\partial t}+\nabla \cdot(\rho \mathbf{u})=0
$$

$\mathrm{f}$ the flow is assumed to be incompressible, (1) can be further simplified as:

$$
\nabla \cdot \mathbf{u}=0
$$

The momentum equation is written as

$$
\frac{\partial(\rho \mathbf{u})}{\partial t}+\nabla \cdot(\rho \mathbf{u u})=-\nabla p+\nabla \cdot(\mu \nabla \mathbf{u})+\rho g+f
$$

where $\rho$ is the density of fluid, $p$ is the pressure filed, $\mathbf{u}$ is the velocity vector, $\mu$ is the dynamic viscosity, and $f$ is the body force apart from gravity, and in this case, is the surface tension of the fluid.

Turbulent governing equations should be included as well to practice the $k-\varepsilon$ model. The equation group is expressed from equation (4) to (5).

$$
\begin{gathered}
\frac{\partial(\rho k)}{\partial t}+\frac{\partial\left(\rho k u_{i}\right)}{\partial x_{i}}=\frac{\partial}{\partial x_{j}}\left(\alpha_{k} \mu_{e f f} \frac{\partial k}{\partial x_{j}}\right)+G_{k}+ \\
G_{b}-\rho \varepsilon-Y_{M}+S_{k} \\
\frac{\partial(\rho \varepsilon)}{\partial t}+\frac{\partial\left(\rho \varepsilon u_{i}\right)}{\partial x_{i}}=\frac{\partial}{\partial x_{j}}\left(\alpha_{\varepsilon} \mu_{e f f} \frac{\partial k}{\partial x_{j}}\right)+C_{1 \varepsilon} \times \\
\frac{\varepsilon}{k}\left(G_{k}+G_{3 \varepsilon} G_{b}\right)-C_{2 \varepsilon} \rho \frac{\varepsilon^{2}}{k}-R_{\varepsilon}+S_{\varepsilon}
\end{gathered}
$$

Gk is the turbulent kinetic energy generated by mean velocity gratitude, and $\mathrm{Gb}$ is that produced by buoyancy. $\alpha \mathrm{k}$ and $\alpha \varepsilon$ are separately reciprocals of Prandtl number of $\mathrm{k}$ and $\varepsilon$, where $\mathrm{k}$ is the turbulent kinetic energy and $\varepsilon$ is the rate of dissipation. YM is the contribution to $\mathrm{k}$ by turbulent momentum. Sk and $\mathrm{S} \varepsilon$ are source terms.

\section{B. VOF method to simulate multiphase flow}

VOF method belongs to the Eulerian-Eulerian model which is the most selected one to calculate multiphase flows [7]. In the VOF functions, $\mathrm{F}$ is defined to represent fractional volume and is decided by equation (6) in a 2-D case [9],

$$
\frac{\partial F}{\partial t}+u \frac{\partial F}{\partial x}+v \frac{\partial F}{\partial y}=0
$$

The interface of different phases are determined by equation (7)

$$
\left\{\begin{array}{l}
F(x, y, t)=1 \text {, the cell is full of liquid } \\
F(x, y, t)=0 \text {, the cell is full of gas } \\
0<F(x, y, t)<1 \text {, the cell contains a part of interface }
\end{array}\right.
$$

Through equation (6) together with basic dynamic flow functions and proper boundary conditions, $F$ field as well as flow field can be solved, and according to equation (7), the free surfaces are determined.

\section{DPM to capture particles}

In the DPM, particles are treated as single micro bodies and one single particle's motion is governed by equation (8) in the Lagrangian coordinates system:

$$
\frac{\mathrm{d} \mathbf{u}_{p}}{\mathrm{~d} t}=F_{D}+\frac{g\left(\rho_{p}-\rho\right)}{\rho_{p}}+F
$$

where

$$
F_{D}=\frac{18 \mu}{\rho_{p} D_{p}^{2}} \frac{C_{D} \mathrm{Re}}{24}
$$

$\mathbf{u}$ is the continuum velocity and $\mathbf{u}_{p}$ is the particle velocity, $\rho$ and $\rho_{p}$ are density of continuum and discrete phase, and $\mu$ is the dynamic viscosity of continuum. $D_{p}$ is the diameter of the particle while $\mathrm{Re}$ is the relative Reynolds number. $C_{D}$ is the drag coefficient decided by experiments [3]. Finally, $F$ consists of effective mass force, force induced by pressure gradient and Brownian force, etc.

During the solution progress, fluid flow fields are calculated at first and every particle's motion are thence gained through equation (8) and (9). Finally, particles distribution is determined according to the motion.

\section{III.EXPERIMENTAL AND NUMERICAL STUDY OF A WATER MODEL}

In this paper, water model experiments are conducted in comparison with the CFD calculations. Only 2-D axisymmetric simulation is performed to decrease the workload. The water model and the corresponding computing mesh, as well as boundary conditions, are shown in Fig .1.

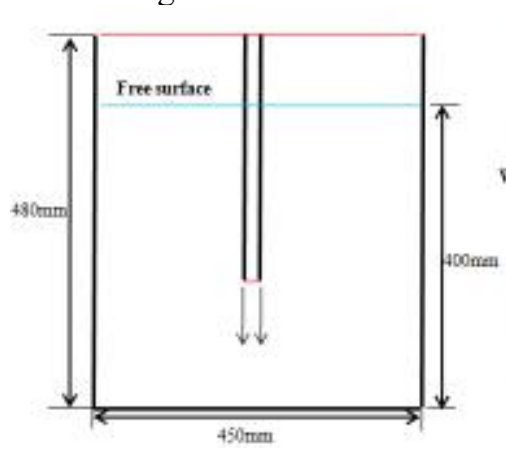

(a)

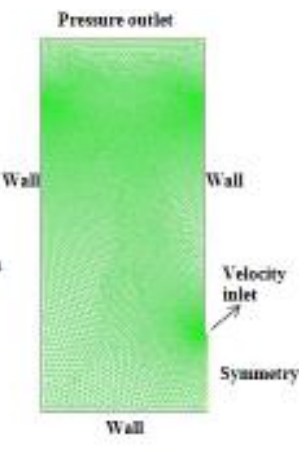

(b)
Figure 1. (a): water model (b): computing mesh

Particle entrance is modeled by injecting a group of particles at the same velocity of the gas at the beginning of each iteration, and following the DPM process, particle motions and distribution are then calculated.

Parameters including lance entering length and inlet velocity are altered and listed in TABLE I to investigate their influence on the multiphase flows and particles evolution.

TABLE I. PARAMETERS OF SIMULATION AND EXPERIMENTS

\begin{tabular}{cccc}
\hline entrance length $(\mathbf{m m})$ & 100 & 200 & 300 \\
\hline inlet velocity $(\mathbf{m} / \mathbf{s})$ & 5 & 10 & 15 \\
\hline
\end{tabular}

Particles distribution is captured by a high-definition camera 1000 frames/s. Photographs at typical instances are shown in Fig .2.

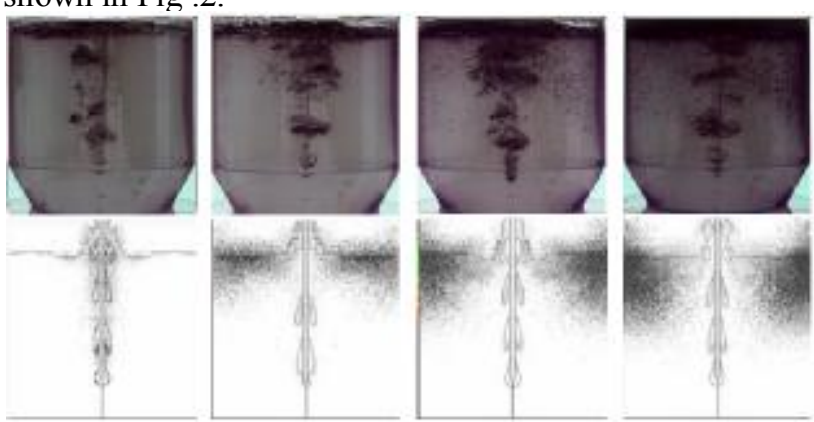

Figure 2. Particles distribution, top: experiment, bottom: simulation. 
Particles distribution is captured by a high-definition camera 1000 frames/s. Photographs at typical instances are shown in Fig .2.

From Fig .2, it can be seen that the particles distribution in experiments and simulation basically agree with each other. Most of the particles floating upward are trapped inside bubbles, and later, due to the buoyancy force, particles are more likely to stay afloat on the free surface rather than inside water. They tend to gather at the wall boundary of the free surface and as it goes deeper, they become sparser.

Traditionally, motions of particles are detected by transition of distributions over time. As for now, with the help of CFD, the flow fields would be easily drawn and even one single particle's trajectory can be recorded [10]. The contrast between fluid velocity field and particles velocity field are shown in Fig .3 and a particle's trajectory is shown in Fig. 4 .

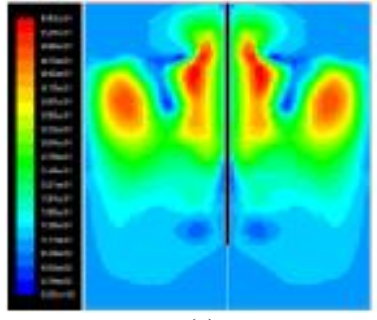

(a)

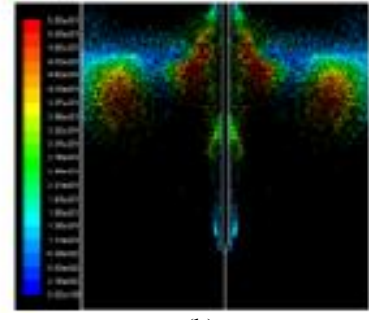

(b)
Figure 3. (a): fluid velocity field (b): particles velocity field

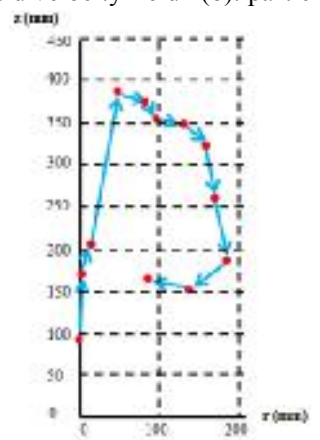

Figure 4. Trajectory of an observed particle.

There is a strong resemblance between the liquid velocity field and discrete particle velocity field according to Fig .3, indicating that particles motions are mainly determined by the fluid flow. Vortices are clearly seen near the free surface which means that the particles afloat on the surface may be sucked into water again, and it explains that particles of smaller density than that of water would gather below the free surface. Also, the trajectory of the particle in Fig .4 illustrates the same pattern.

To summarize, the motion of particles injected by the immersed lance into a water tank can be describe as follows. Particles are first trapped inside bubbles, and thanks to the buoyancy force, they start going upward. As the gas plumes collapse during floating and as particles penetrate through bubbles, they become in direct contact with water. At the top free surface of the tank, every particle is set free from bubbles and they begin to travel horizontally with tiny-scale vertical oscillation until they arrive at the side walls. Finally, with the strong vortex flow near the surface, particles are sucked into water and cyclically travel up and down.
In desulphurization processes, one of the most considered problems is to detect maximum depth that particles near the side wall can arrive, which denotes active zone in molten iron where desulphurization is effective. According to the particle tracking function, this problem is conveniently solved. Referring to table 1 , the maximum sinking depth is gained for every case and is shown in Fig .5.

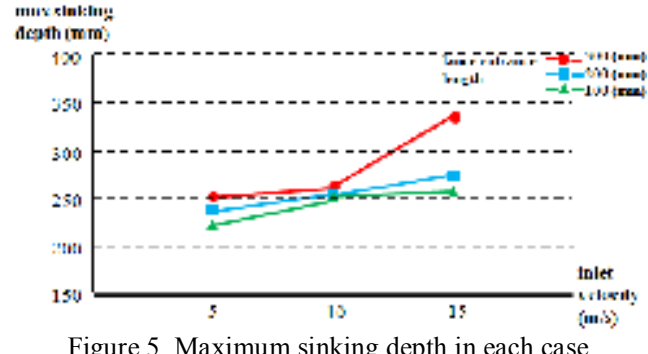

It can be seen that with the lance set deeper into the fluid, the maximum sinking depth of particle grows and as the inlet velocity rises, the depth becomes greater. Nonetheless, when the injection velocity is relatively low, growth in lance entering length would make no big difference. As for great enough inlet speed, maximum sinking depth increases substantially. It can be explained that higher injection velocity brings in greater stirring energy and deeper lance means more time for bubble to travel through liquid phase which makes the agitation last longer.

\section{IV.NUMERICAL STUDY OF THE DESULPHURIZATION PROCESS}

In section III, a qualitative agreement between CFD results and experimental results is arrived, and due to the inconvenience of conducting experiment in real metallurgy ladle, only CFD calculation is performed to simulate desulphurization process. The molten iron physical parameters are named in TABLE II, in comparison with that of water. The ladle model and computing mesh are shown in Fig .6. Attention should be paid that numerical model of ladle is defined without using symmetry laws.

TABLE II. PHYSICAL PARAMETERS OF MOLTEN IRON AND WATER

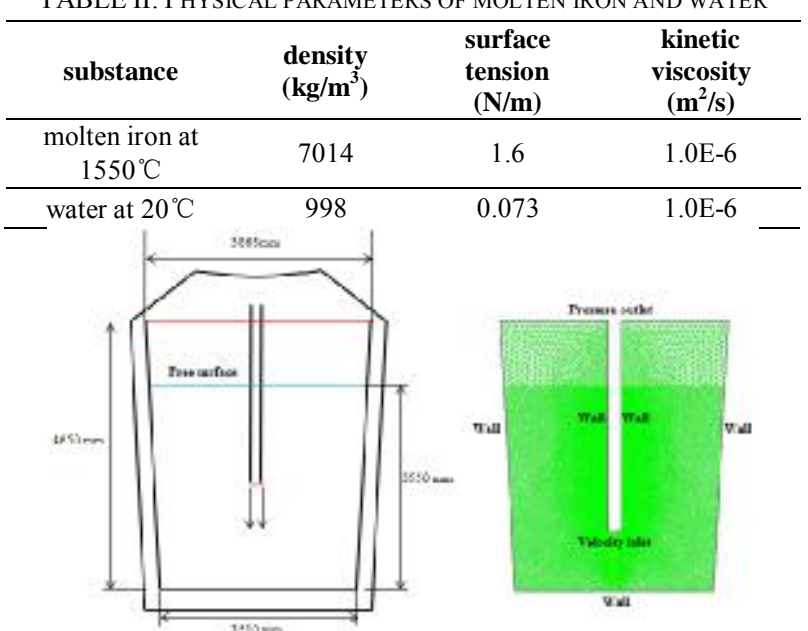

(a)

(b)

Figure 6. (a): ladle model (b): computing mesh

CFD results are shown in typical instances in Fig .7. 

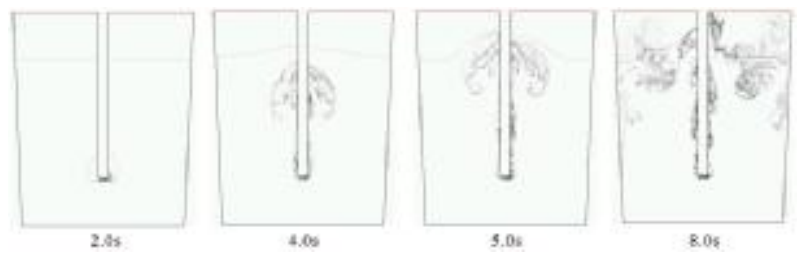

Figure 7. Typical instances of multiphase flow in a ladle by CFD simulation

From Fig .7, transformations of bubbles and particles distribution are clearly seen. At first, particles are trapped in bubbles of inert gas traveling upward. During this process, most of particles stay inside bubbles without contact to molten iron. As the bubbles reach the free surface, they began to collapse, and particles are set free. Due to the stirring effect of bubbles, particles flow towards the side walls with vortex motion and they began sinking into molten iron again. Most of them tend to gather near the side wall boundary of free surface.

\section{CONCLUSIONS}

From the numerical and experimental studies in this paper, the following conclusions are drawn.

1. The results of the CFD algorithm agree with experimental results of water model qualitatively on multiphase flow patterns and particles distribution.

2. Before particles arrive at the free surface, most of them are trapped in bubbles and move upward with the bubbles. After the arrival, most of particles travel with the liquid vortex flow towards side walls and tend to stay near the side wall boundary of the free surface.

3. The maximum sinking depth of particles near side wall boundaries grows as the injection speed and lance entering length increase. When the injection velocity is relatively low, growth in lance entering length makes small differences. However, at large enough inlet speeds, the maximum sinking depth increases substantially when lance entering length grows.

\section{REFERENCES}

[1] Sahai Y, Guthrie R I L. Recent advances in the hydrodynamics of metallurgical processing $[\mathrm{J}]$. Advances in transport processes.(eds. Mazumdar, AS; Mashelakar, RA), 1986: 1-48. 、

[2] Sinha U P, McNallan M J. Mixing in ladles by vertical injection of gas and gas-particle jets-A water model study [J]. Metallurgical and Materials Transactions B, 1985, 16(4): 850-853.

[3] OZAWA Y, MORI K. Critical condition for penetration of solid particle into liquid metal $[\mathrm{J}]$. Transactions of the Iron and Steel Institute of Japan, 1983, 23(9): 769-774.

[4] Torii S, Yang W J. Melt-particle mixing in gas-stirred ladles with throughflow [J]. Experiments in fluids, 1992, 13(1): 37-42.

[5] Morud K E, Hjertager B H. LDA measurements and CFD modelling of gas-liquid flow in a stirred vessel [J]. Chemical Engineering Science, 1996, 51(2): 233-249.

[6] Mazumdar D, Guthrie R I L. Numerical computation of flow and mixing in ladle metallurgy steelmaking operations (CAS method) [J]. Applied mathematical modelling, 1986, 10(1): 25-32.

[7] Venturini G, Goldschmit M B. Gas-liquid reaction model in gasstirred systems: Part 1. Numerical model [J]. Metallurgical and Materials Transactions B, 2007, 38(3): 461-475.

[8] Xia J L, Ahokainen T, Holappa L. Analysis of flows in a ladle with gas - stirred melt [J]. Scandinavian journal of metallurgy, 2001, 30(2): 69-76.

[9] Hirt C W, Nichols B D. Volume of fluid (VOF) method for the dynamics of free boundaries $[\mathrm{J}]$. Journal of computational physics, 1981, 39(1): 201-225.

[10] Fluent I N C. Fluent 6.3 Users Guide[J]. Fluent documentation, 2006. 